\title{
EOPS: ¿Cuál es el beneficio del tiotropio en pacientes con enfermedad pulmonar obstructiva crónica?
}

Wich is the benefit of tiotropium in patients with chronic obstructive pulmonar disease

Patricia Aruj ${ }^{\star}$

\begin{abstract}
Resumen
Las recomendaciones vigentes para el tratamiento de los pacientes con enfermedad pulmonar obstructiva crónica (EPOC) indican que los fármacos anticolinérgicos desempeñan una función imporatante. El desarrollo de fármacos anticolinérgicos seguros, efectivos, con una selectividad funcional relativa para los subtipos de receptores muscarínicos ha generado un renovado interés por el tratamiento broncodilatador anticolinérgico. En este artículo se plantea la ventaja relativa de la sustitución de ipratropio con tiotropio en un paciente con EPOC severa e internaciones frecuentes que era medicado con ipratropio, budesonide y formoterol sin observarse mejoría clínica. Se concluye que la sustitución puede tener beneficios en la disminución de las exacerbaciones e internaciones así como en la calidad de vida de estos pacientes.
\end{abstract}

\section{Abstract}

Current recommendations for treatment of patients with chronic obstructive pulmonar disease (COPD) show that anticholinergics play an imoprtant role. The development of safe, effective anticholinergics drugs, with a relative functional selectivity for different muscarinic receptors subtypes has renowned the interest for the anticholinergic bronchodilator treatment. This article discuss the potential advantage of the replacement of ipratropium for tiotropium in a patient with severe COPD and frequent hospitalizations that was receiving ipratropium, budesonide and formoterol without noticing clinical benefits. It is concluded that the substitution can have benefits in reducing exacerbations and hospitalizations as well as improving patients'quality of life.

Palabras clave: enfermedad pulmonar obstructiva crónica, tiotropio, ipratropio, agonistas beta. Key words: chronic obstructive pulmonary disease, tiotropium, ipratropium, beta agonists.

Aruj P. Evidencia orientada al paciente. ¿Cuál es el beneficio del tiotropio en pacientes con enfermedad pulmonar obstructiva crónica? Evid. actual. práct. ambul; 11(4):126-127, Jul-Ago.2008.

\section{Escenario clínico}

Se trata de un paciente de 65 años con diagnóstico de EPOC severa e internaciones frecuentes por exacerbación. Ex tabaquista hasta hace dos años, de 40 paquetes/años. Última internación en los tres meses previos con un volumen espiratorio forzado en el primer segundo (VEF1) posterior estable de $1200 \mathrm{~mL}(48 \%$ del esperable) con espirometrías previas con VEF1 de $60 \%$. Tiene disnea habitual en clase funcional II, sin requerimiento de oxígeno y una tomografía de tórax con enfisema centrolobulillar bilateral. Luego del alta de su última internación se agrega al tratamiento habitual (bromuro de ipratropio, dos disparos tres veces por día) budesonide más formoterol cada 12 horas. Luego de una mejoría inicial el paciente refiere dificultad progresiva en sus actividades laborales diarias. Se plantea si la sustitución del bromuro de ipratropio por tiotropio sería beneficiosa.

\section{Pregunta que generó el caso}

En los pacientes EPOC (población), ¿la administración de tiotropio (intervención) en comparación con el bromuro de ipratropio (comparación), enlentece la progresión del deterioro de la función pulmonar, aumenta la calidad de vida y/o reduce el número de exacerbaciones (resultados)?

\section{Estrategia de Búsqueda}

Se realizó una búsqueda en Pubmed y en Tripdatabase empleando como palabras clave "Chronic Obstructive Pulmonary Disease" AND tiotropium OR ipratropium limitando a revisiones sistemáticas o meta-análisis de menos de cinco años de publicación. Se hallaron 19 citas, de las cuales se seleccionó una revisión Cochrane como la más adecuada para responder la pregunta.

\section{Contexto clínico}

Las recomendaciones vigentes para el tratamiento de los pacientes con EPOC indican que los fármacos anticolinérgicos desempeñan una función prominente ${ }^{1}$. De modo similar al ipratropio, el tiotropio se une a los receptores muscarínicos M1, M2 y $M 3$, pero a diferencia de este que tiene actividad selectiva para estos receptores, el tiotropio se disocia lentamente de los receptores $\mathrm{M} 1$ y $\mathrm{M} 3$, pero rápidamente de los receptores $\mathrm{M}^{2}{ }^{2}$.
La disociación lenta de los receptores M1 y M3 produce un efecto broncodilatador que se mantiene durante más de 24 horas, lo que permite usar una sola dosis diaria ${ }^{3}$. La disociación rápida de los receptores $\mathrm{M} 2$ también puede ser ventajosa, ya que son receptores inhibitorios por retroalimentación. Por consiguiente, el bloqueo de los receptores M2 aumenta paradójicamente la descarga de acetilcolina, mientras que el desbloqueo la disminuye ${ }^{4}$. En consecuencia, el tiotropio podría tener beneficios clínicos sobre el ipratropio, uno de los tratamientos de primera línea actuales de la EPOC estable.

Sin embargo, las guías actuales para el tratamiento de la EPOC $^{5}$ indican que:

En pacientes en estadío I (leves con $\mathrm{VEF}_{1}$ mayor a $80 \%$ ) pueden beneficiarse con el uso de un broncodilatador (BD) de acción corta a demanda para controlar la disnea. Si no se dispone de $\mathrm{BD}$ inhalados podría considerarse el uso de teofilina.

En pacientes en estadío II (moderados: $\mathrm{VEF}_{1}$ menor a $80 \%$ y mayor a $50 \%$ ) a IV (severos y muy severos: VEF 1 menor a $50 \%$ ) cuya disnea durante las actividades de la vida diaria no mejora con $\mathrm{BD}$ de acción corta a demanda se recomienda agregar un BD de acción prolongada (recomendación tipo A). Sin embargo, hay insuficiente evidencia en favor de uno $u$ otro BD de acción prolongada. En pacientes con regular uso de BD de acción prolongada que necesitan mayor control de los síntomas, agregar teofilina puede producir beneficios adicionales (recomendación tipo B). En pacientes severos con una historia de repetidas exacerbaciones (tres o más en los últimos tres años) el tratamiento regular con corticoides inhalados reduce la frecuencia de las exacerbaciones y mejora la calidad de vida pudiendo usarse en combinación con BD acción prolongada. No se recomienda el uso de corticoides vía oral. La vacuna antigripal reduce las enfermedades serias y la mortalidad en el EPOC en un $50 \%$.

\section{Resumen de la evidencia}

Barr RG, Bourbeau J, Camargo CA, Ram FSF. Tiotropio inhalado para la enfermedad pulmonar obstructiva crónica estable (Revisión Cochrane traducida). Biblioteca Cochrane Plus, 2007 Número 2. Oxford: Update Software Ltd. Disponible en: http://www.update-software.com. (Traducida de The Cochrane Library, 2007 Issue 2. Chichester, UK: John Wiley \& Sons, Ltd.). Fecha de la modificación más reciente: 31 de enero de 2005. 


\section{Objetivo}

Evaluar la eficacia del tiotropio sobre las variables principales de evaluación clínica, las escalas de calidad de vida y de síntomas y sobre la función pulmonar a partir de los datos disponibles de ensayos clínicos que comparen el tiotropio con placebo, bromuro de ipratropio y agonistas 32 de acción prolongada.

\section{Estrategia de búsqueda}

Se buscó en las siguientes bases de datos: CENTRAL, MEDLINE, EMBASE y CINAHL. La fecha de la última búsqueda fue octubre 2004.

\section{Criterios de inclusión}

Ensayos clínicos aleatorizados (ECA) que hubieran comparado tiotropio con placebo, bromuro de ipratropio, o agonistas $B 2$ de acción prolongada. Como el propósito principal de la revisión era evaluar las respuestas clínicas a largo plazo al tiotropio, se excluyeron los estudios que siguieron a los pacientes durante menos de un mes.

Se incluyeron adultos mayores de 35 años con EPOC conocida, estable, según los criterios diagnósticos de la American Thoracic Society (ATS), la European Respiratory Society (ERS) o los criterios diagnósticos GOLD. Se hicieron comparaciones entre la administración de tiotropio versus placebo; bromuro de ipratropio agonistas 32 de acción prolongada (salmeterol o formoterol).

Como medidas de resultados primarios se buscaron criterios clínicos, exacerbaciones, hospitalizaciones y mortalidad; y como resultados secundarios, escalas de calidad de vida, puntuación autocalificada de los síntomas de disnea, cambio del $V_{E F}$ y cambio de la capacidad vital forzada (CVF) al inicio y en el estado estable ( 8 a 15 días después del comienzo del tratamiento) rendimiento en el ejercicio (caminata de seis minutos) uso de medicación de rescate durante el período de tratamiento y otros fármacos, incluso antibióticos y esteroides; eventos adversos (palpitaciones, sequedad bucal, visión borrosa, obstrucción urinaria y constipación).

\section{Resultados principales}

De las 69 referencias identificadas, nueve ECA (6584 pacientes) cumplieron los criterios de inclusión. El tiotropio disminuyó la chance de una exacerbación del EPOC (OR 0,74; IC95\% 0,66 a 0,83) y de las hospitalizaciones relacionadas (OR 0,64 ; IC95\% 0,51 a 0,82) en comparación con placebo o ipratropio. Al aplicarse esta reducción a un riesgo inicial anual de $45 \%$ para las exacerbaciones y de $10 \%$ para la hospitalización, el número de pacientes necesarios a tratar con tiotropio durante un año fue 14 (IC95\%11 a 22) para prevenir una exacerbación y 30 (IC95\% 22 a 61) para prevenir una hospitalización en relación al placebo o ipratropio. No se observaron diferencias estadísticamente significativas cuando se lo comparó con los agonistas 32 de acción prolongada. Fueron evidentes patrones similares en las escalas de calidad de vida y de síntomas. Los aumentos del VEF y la CVF comparados con el valor inicial fueron significativamente mayores con tiotropio que con placebo, ipratropio o agonistas $B 2$ de acción prolongada durante 6 a 12 meses. La disminución al año del VEF medi- $_{1}$ do en el estado estable fue de $30 \mathrm{~mL}$ (IC95\% 7 a $53 \mathrm{~mL}$ ) menor con tiotropio que con placebo o con ipratropio; y no se encontraron datos sobre la disminución del VEF 1 en el estado estable para los agonistas 32 de acción prolongada. La sequedad bucal aumentó con tiotropio.

\section{Conclusión de los autores}

Comparado con placebo e ipratropio, el tiotropio disminuyó las exacerbaciones de la EPOC y las hospitalizaciones relacionadas. También mejoró la calidad de vida relacionada con la salud y las puntuaciones de los síntomas en los pacientes con enfermedad moderada y grave y puede haber hecho más lenta la disminución del VEF1. Se requieren estudios a largo plazo adicionales para evaluar su efecto sobre la mortalidad y el cambio del VEF 1 , para aclarar su función en comparación con, o en combinación con agonistas 32 de acción prolongada y para evaluar su efectividad en la EPOC leve y muy grave.

\section{Comentario}

Esta revisión sistemática sobre el uso de tiotropio para el EPOC estable demostró disminución de las exacerbaciones y las hospitalizaciones relacionadas al compararlo con placebo o ipratropio. Se observaron mejorías similares en las escalas de calidad de vida y de síntomas. Los aumentos del VEF 1 y de la CVF con relación al valor inicial fueron significativamente mayores con tiotropio que con placebo, ipratropio y con los agonistas $B 2$ de acción prolongada. Aunque el número total de pacientes que contribuyeron con datos de un año de seguimiento en estos ensayos fue escaso, hubo una diferencia estadísticamente significativa de la disminución del VEF 1 mínima en el estado estable con tiotropio en comparación con placebo o ipratropio.

Los beneficios observados con tiotropio para las exacerbaciones y hospitalizaciones relacionadas fueron grandes y clínicamente importantes. Las exacerbaciones de la EPOC fueron responsables de ocho millones de visitas de pacientes ambulatorios, de 1,5 millones de emergencia y de 726.000 hospitalizaciones en EE.UU. durante $2000^{6}$. La disminución de un tercio de las hospitalizaciones podría potencialmente producir disminuciones significativas de la morbilidad y del costo. Aunque el costo de la droga sea mayor al del ipratropio (siete veces); el tiotropio demostró ser costo-efectivo en EPOC moderado a severo ${ }^{7}$. Los cambios en la calidad de vida, en las escalas de los síntomas, en los índices espirométricos y en las exacerbaciones fueron clínicamente significativos, cuando se lo comparó con placebo y con ipratropio, cambios también observados en otros estudios ${ }^{8,9}$.

Debido a la falta de medicación que logre modificar el curso de la enfermedad o el pronóstico, la alternativa de asociar tiotropio al tratamiento de mantenimiento del EPOC moderado a severo podría tener lugar en las recomendaciones actuales ${ }^{10}$.

\section{Referencias}

1. Ferguson G y col. Management of chronic obstructive pulmonary disease. N Engl J Med 1993;(328):1017-22

2. Haddad $\mathrm{E}$ y col. Characterization of $3 \mathrm{H} \mathrm{Ba} 679 \mathrm{BR}$, a slowly dissociation muscarinic antagonist, in human lung: radioligand binding and autoradiographic mapping. Mol Pharmacol 1994;(45):899-907.

3. Disse B y col. Tiotropium (Spiriva): mechanistical considerations and clinical profile in obstructive lung disease. Life Sci 1999;(64):457-64.

4. Barnes P. The pharmacological properties of tiotropium. Chest 2000;117(2) Suppl:63S-66S.

5. Global Strategy for the Diagnosis, Management, and Prevention of Chronic Obstructive Pulmonary Disease (2006).

6. Mannino D y col. Chronic obstructive pulmonary disease surveillance United States, 1971-2000.

7. Oostenbrink J y col. Eur Respir J 2004;23:241-9.

8. Stanbrook M. Review: tiotropium reduces exacerbations and hospital admissions in COPD and improves quality of life. Evid. Based Med. 2005; 10; 176

8. Stanbrook M. Review: tiotropium reduces exacerbations and hospital admissions in COPD and improves quality of life. Evid. Based
9. Sin D y col. Contemporary management of chronic obstructive pulmonary disease: scientific review. JAMA 2003; 290: 2301-12.

10. van Noord J y col. A randomized controlled comparison of tiotropium and ipratropium in the treatment of chronic obstructive pulmonary disease. Thorax $2000 ; 55,289-294$. 